\title{
Análisis estilístico del habla de Mr. Grimwig en Oliver Twist, de Charles Dickens, y su traducción al español
}

\author{
(Stylistic Analysis of Mr. Grimwig's Speech in Charles \\ Dickens's Oliver Twist and Its Translation into Spanish)
}

\author{
Pablo Ruano San Segundo \\ pauruano@unex.es \\ Universidad de Extremadura
}

Fecha de recepción: 31 de mayo de 2016

Fecha de aceptación: 28 de junio de 2016

Resumen: En este artículo se lleva a cabo un análisis traductológico del habla de Mr. Grimwig en Oliver Twist, de Charles Dickens, en las versiones españolas de Alfredo Yáñez, Vergara y José Méndez Herrera. El objetivo del estudio consiste en medir el grado de acierto de estas tres traducciones en el traslado al español de un aspecto de importancia cardinal en el estilo dickensiano: la memorabilidad de sus personajes como recurso de caracterización en el marco de la novela por entregas decimonónica inglesa. Con apariciones aisladas en únicamente cuatro capítulos de la historia, Mr. Grimwig constituye un ejemplo paradigmático de las técnicas de caracterización del autor, pues es acreedor de un habla idiosincrásica que le individualiza y facilita su reconocimiento en el transcurso de la historia. En este artículo se analiza en qué medida esa habla es producto de la publicación fasciculada original de la novela, así como el modo en el que los traductores españoles la trasladan al español, de tal suerte que pueda comprobarse si las versiones objeto de estudio -y en caso de que así sea, hasta qué punto- conservan uno de los marchamos estilísticos más representativos del autor victoriano.

Palabras clave: Dickens, Mr. Grimwig, Individualización, Habla, Traducción.

Abstract: This article presents a translation analysis of Mr. Grimwig's speech in Charles Dickens's Oliver Twist in the Spanish versions of Alfredo Yáñez, Vergara and José Méndez Herrera. The aim of the study is to gauge the degree of 
faithfulness of these three translations in rendering into Spanish one of Dickens's best-known stylistic features: his characters's memorability as a typical characterizing device of nineteenth-century English serialized fiction. With appearances in only four chapters of the story, Mr. Grimwig constitutes a paradigmatic example of Dickens's celebrated techniques of characterization. This character possesses an idiosyncratic way of speaking, which singles him out and helps the reader to recognize him in the course of the story. In this article, I will analyze if this way of speaking is a stylistic outcome of the serialized mode of publication in which Oliver Twist was originally released and how translators render it into Spanish. Thus, it will be possible to conclude whether and to what extent the three versions under analysis here maintain one of the Victorian author's most distinguished stylistic features.

Keywords: Dickens, Mr. Grimwig, Individualization, Speech, Translation.

\section{INTRODUCCIÓN}

En el presente artículo se lleva a cabo un análisis traductológico del habla de Mr. Grimwig en Oliver Twist, segunda novela de Charles Dickens, en las versiones españolas de mediados del siglo XX de Alfredo Yáñez (1996 [1945]), Vergara (1970 [1965]) y José Méndez Herrera (2003 [1948]). Mr. Grimwig constituye un ejemplo paradigmático de las célebres técnicas de caracterización del autor victoriano en el marco de la novela por entregas decimonónica inglesa. Su alto grado de individualización, logrado a través de un habla muy peculiar que facilita su reconocimiento por parte del lector en sus intervenciones dispersas en distintos puntos de la historia, le convierte en un ejemplo ideal para analizar el nivel de fidelidad de los traductores españoles al trasladar uno de los marchamos estilísticos más representativos del autor. El artículo se divide en dos partes. En primer lugar, se realiza un análisis del habla de Mr. Grimwig en la novela, de tal suerte que pueda demostrarse su valor estilístico como recurso de caracterización. A continuación, se evalúa la forma en que los tres traductores trasladan esta habla al español. Este análisis servirá no solo para medir la calidad de las tres versiones objeto de estudio, sino para demostrar la necesidad de no descuidar aspectos clave del texto inglés, producto de un estilo claramente influido por las circunstancias originales de su publicación. Las diferencias que existen en las tres versiones ilustrarán, además, las implicaciones que acarrea la diferente gestión de la traducción de uno de los rasgos distintivos del estilo dickensiano y demostrará la importancia de conservarlo en el texto de llegada. 


\section{EL HABLA DE MR. GRIMWIG}

La relevancia de Mr. Grimwig en la trama argumental de Oliver Twist es prácticamente nula. Sus apariciones en la historia, de hecho, se limitan a cuatro capítulos - véase tabla 1- en los que Dickens lo utiliza como contrapunto del bondadoso Mr. Brownlow. Su contribución en la historia es, grosso modo, la de poner en tela de juicio los buenos propósitos del pequeño Oliver e intentar hacer ver a su amigo Mr. Brownlow que las esperanzas que tiene depositadas en el joven son inútiles. A pesar de no tener razones para desconfiar del muchacho, su carácter suspicaz -que en realidad esconde un buen corazón- hace que le resulte muy difícil creer que alguien con ese aspecto pueda tener intenciones nobles. Por el contrario, cuando en el capítulo 14 Mr. Brownlow envía a Oliver a devolver unos libros, Mr. Grimwig le asegura que teniendo "un traje nuevo, un montón de libros de valor debajo del brazo y un billete de cinco libras en el bolsillo" (Méndez Herrera, 2003 [1948]: 348), Oliver se irá "en busca de sus antiguos amigos, los rateros, y se reirá de vos [léase Mr. Brownlow]" (ibid.). Que Oliver acabe siendo capturado por Sikes y no pueda regresar, como había prometido a Mr. Brownlow, desde luego alimenta el recelo de Grimwig hacia el joven. En el capítulo 41, cuando Oliver se vuelve a encontrar con Mr. Brownlow y le cuenta lo ocurrido, Mr. Grimwig reconoce que el muchacho en realidad no es un truhan, aunque su terquedad le hace mantener que Oliver no regresó como había prometido y que, al menos en esta parte, tenía razón sobre el joven.

Sin embargo, a pesar de su poca transcendencia y de su aparición en únicamente cuatro de los cincuenta y tres capítulos que conforman la novela, Dickens no descuida su caracterización. Por el contrario, al tratarse de un personaje que únicamente interviene en momentos aislados de la novela, el autor lo perfila de un modo muy particular para que el lector no tenga dificultades en identificarlo cada vez que aparece. Esta es una de las marcas estilísticas dickensianas por excelencia, que consiste en hacer de sus personajes con menos apariciones criaturas fácilmente reconocibles en el transcurso de la publicación fasciculada (Ingham, 1979: 144) ${ }^{1}$. Se trata de un recurso que gozaba de gran popularidad entre el público de la época, pues existía un componente de congratulación por parte del lector en el reconocimiento de las particularidades del personaje tras varias entregas ausente (Brook, 1970: 144). En el caso concreto de Mr. Grimwig, los rasgos que más sobresalen de su carácter son su irascibilidad y su testarudez. Estos se hacen patentes, por encima de todo, a

${ }^{1}$ Oliver Twist se publicó, en entregas mensuales, entre febrero de 1837 y abril de 1839 (Vann, 1985: $62)$. 
través de un habla idiosincrásica, con unas particularidades discursivas muy marcadas. Esta forma de hablar, como es tradición en la novela victoriana, se construye a través de la estrategia de estilo directo (Busse, 2010: 90), capaz de cumplir con las exigencias de dramatización y caracterización que requiere el género novelesco (Semino y Short, 2004: 89). Esta estrategia de representación del discurso se construye sobre dos bloques bien diferenciados: las palabras del personaje y el narrador que las introduce. Por ejemplo:

(1) 'A bad one! I'll eat my head if he is not a bad one,' growled Mr. Grimwig, speaking by some ventriloquial power, without moving a muscle of his face.

(Dickens, 2008 [1837-1839]: 331)

De un lado, las palabras del personaje son la parte más llamativa en lo que a la representación del habla se refiere. En ellas se advierten algunas de las técnicas de caracterización por las que Dickens goza de más reconocimiento, como el uso de dialectos ${ }^{2}$, las particularidades en el habla de los personajes ${ }^{3} 0$ las muletillas (Golding, 1985). Una muletilla es, de hecho, la marca discursiva que caracteriza e individualiza el habla de Mr. Grimwig. Sus constantes amenazas de comerse, literalmente, la cabeza ("I'll eat my head if he is not a bad one" en el caso del ejemplo anterior) no solo le dotan de comicidad, sino que facilitan su identificación a lo largo de la novela, pues, efectivamente, se trata del "magnífico ofrecimiento con el que el señor Grimwig apoyaba y confirmaba todos sus asertos" (Méndez Herrera, 2003 [1948]: 345). Esta expresión contribuye, además, a ilustrar su carácter iracundo y pertinaz.

De otro lado, las palabras del narrador introduciendo el discurso del personaje, aunque son menos llamativas, resultan igualmente significativas desde un punto de vista estilístico. De hecho, en la proyección del habla de los personajes por parte del narrador en las novelas dickensianas se advierten patrones que contribuyen a la caracterización de las figuras que pueblan su universo ficticio. Estos patrones tienen que ver, sobre todo, con el lenguaje gestual y con el modo de articulación de las palabras (cf. Mahlberg, 2013; Ruano

${ }^{2}$ Como ejemplos paradigmáticos del uso de dialectos por parte de Dickens para caracterizar a sus personajes puede señalarse a Sam Weller (Pickwick Papers) o a Sarah Gamp (Martin Chuzzlewit), dos de sus personajes más recordados debido, en gran medida, a su uso de la variedad Cockney (cf. Page, 1970).

${ }^{3}$ En cuanto a las particularidades discursivas de algunos de los personajes cabe mencionar el ceceo de Sleary, el gerente del circo de Hard Times (Hawes, 2002: 215) Desde su primera aparición, Dickens construye el discurso del personaje de tal manera que el ceceo que le caracteriza sea el aspecto que más llame la atención del lector cuando habla. 
San Segundo, 2016). En el caso de Mr. Grimwig, varias de sus intervenciones aparecen introducidas, como en el ejemplo anterior, por el verbo growl. El empleo repetido de esta forma verbal, como se podrá comprobar a continuación, contribuye a proyectar su carácter irascible.

En suma, tanto las palabras de Mr. Grimwig como la proyección de estas por parte del narrador contienen rasgos que contribuyen a caracterizar al viejo solterón a través de su habla. Esta forma de caracterización, como cabría esperar, no es exclusiva de Mr. Grimwig en Oliver Twist, sino que constituye uno de los sellos distintivos del estilo de Dickens. De hecho, las hablas idiosincrásicas de los personajes suelen considerarse uno de los recursos de caracterización más efectivos empleados por el autor en el marco de la publicación fasciculada en que desarrolló su producción, pues permiten a los lectores reconocer a los personajes tras varias entregas ausentes de la trama argumental (Fludernik, 2009: 73). En el caso concreto de Mr. Grimwig, sus constantes amenazas con comerse la cabeza y la proyección de su discurso a través de growl comportan, debido a su disposición estratégica en momentos clave de sus intervenciones, un ejemplo paradigmático de las técnicas de caracterización de Dickens en este sentido, siendo un caso de estudio ideal para medir el grado de fidelidad de las traducciones españolas de uno de los marchamos estilísticos más señeros del autor victoriano.

\subsection{Análisis de "I'll eat my head" y growl como recursos de caracterización}

Para comprender la importancia de traducir tanto la muletilla como el verbo de habla empleados por Dickens para construir el habla de Mr. Grimwig en Oliver Twist es necesario, en primer lugar, analizar el valor estilístico de estos recursos a lo largo de las apariciones del personaje en la novela. En la siguiente tabla se muestran los cuatro capítulos en los que el viejo solterón interviene, así como las entregas en las que se encuentran y la fecha original de publicación de estas:

\begin{tabular}{|c|c|c|}
\hline \multicolumn{3}{|c|}{ Mr. Grimwig } \\
\hline Capítulo & Entrega & Fecha de publicación \\
\hline Capítulo 14 & 7 & Septiembre 1837 \\
\hline Capítulo 17 & 8 & Noviembre 1837 \\
\hline Capítulo 41 & 18 & Octubre 1838 \\
\hline
\end{tabular}

\footnotetext{
${ }^{4}$ Para un desglose pormenorizado de la publicación original de la novela según capítulos, entregas y fechas, véase Vann (1985: 62).
} 


\begin{tabular}{|l|l|l|}
\hline Capítulo 51 & 22 & Febrero 1839 \\
\hline
\end{tabular}

Tabla 1. Capítulos en los que interviene Mr. Grimwig en Oliver Twist

Como se puede observar, la presencia textual del personaje es muy intermitente y está marcada por largas ausencias, no solo en términos capitulares, sino también en lo que al espacio de tiempo entre la publicación de las entregas se refiere. Entre la primera y la segunda intervención transcurren dos meses ${ }^{5}$; desde la octava hasta la decimoctava transcurren once; y desde esta última hasta la vigesimosegunda, otros cuatro. Tales ausencias resultan fundamentales para comprender por qué Dickens construye el habla del personaje de la manera en que lo hace, pues tanto los usos de la muletilla como los del verbo de habla aparecen en momentos clave de la vida textual del personaje, como se detalla a continuación.

Los primeros usos de la muletilla, por ejemplo, se localizan en el capítulo 14. De hecho, Dickens la utiliza en dos ocasiones en sus dos primeras intervenciones en la historia, aprovechando además para informarnos, a través de la figura del narrador, de que se trata del hábito lingüístico más característico del personaje. He aquí el extracto de la novela donde aparecen estos tres ejemplos ${ }^{6}$ :

(2) 'Look here! do you see this! Isn't it a most wonderful and extraordinary thing that I can't call at a man's house but I find a piece of this poor surgeon's friend on the staircase? l've been lamed with orange-peel once, and I know orange-peel will be my death, or I'll be content to eat my own head, sir!'

This was the handsome offer with which Mr. Grimwig backed and confirmed nearly every assertion he made; and it was the more singular in his case, because, even admitting for the sake of argument, the possibility of scientific improvements being brought to that pass which will enable a gentleman to eat his own head in the event of his being so disposed, Mr. Grimwig's head was such a particularly large one, that the most sanguine man alive could hardly entertain a hope of being able to get through it at a sitting - to put entirely out of the question, a very thick coating of powder.

\footnotetext{
${ }^{5}$ A pesar de tratarse de entregas consecutivas (véase tabla 1), entre la publicación de ambas transcurren dos meses, ya que en octubre de 1837 no salió a la venta el número correspondiente. En su lugar, Dickens publicó un informe de la Mudfog Association of the Advancement of Everything (Vann, 1985: 62).

${ }^{6}$ La negrita, al igual que en el resto de ejemplos del artículo, es mía.
} 
I'll eat my head, sir,' repeated Mr. Grimwig, striking his stick upon the ground. 'Hallo! What's that!' looking at Oliver, and retreating a pace or two. (Dickens, 2008 [1837-1839]: 105-106)

Estos ejemplos se ven reforzados por otros dos usos de la muletilla por parte del personaje justo a continuación:

(3) 'You don't mean to say that's the boy who had the fever, I hope?' said Mr. Grimwig, recoiling a little more. 'Wait a minute! Don't speak! Stop-' continued Mr. Grimwig, abruptly, losing all dread of the fever in his triumph at the discovery; 'that's the boy who had the orange! If that's not the boy, sir, who had the orange, and threw this bit of peel upon the staircase, l'll eat my head, and his too.' (Dickens, 2008 [1837-1839]: 106)

(4) 'No,' he said, smiting the table with his fist, 'I do not. The boy has a new suit of clothes on his back, a set of valuable books under his arm, and a fivepound note in his pocket. He'll join his old friends the thieves, and laugh at you. If ever that boy returns to this house, sir, I'll eat my head.' (Dickens, 2008 [1837-1839]: 110)

Como se puede observar, nada más presentarlo, Dickens incide en la característica que más descuella de Mr. Grimwig, introduciéndola en su primera intervención, informándonos de ella a renglón seguido y reincidiendo en su uso en sucesivos actos de habla del personaje. Como se ha apuntado, este afán de marcar al personaje con la particularidad que lo caracteriza es una de las idiosincrasias dickensianas por antonomasia, fruto del deseo de grabar en la mente del lector la imagen del personaje para que este pueda reconocerlo en el transcurso de la novela.

En la siguiente entrega, en el capítulo 17, Mr. Grimwig vuelve a aparecer, haciendo nuevamente uso de la muletilla en su primera intervención tras tres capítulos ausente. He aquí el ejemplo:

(5) 'A beadle. A parish beadle, or l'll eat my head.' (Dickens, 2008 [18371839]: 135)

Desde luego, resulta llamativo que sea en su primera intervención en el capítulo cuando el personaje haga uso de la muletilla y no en otro momento del episodio. Su empleo justo cuando el lector vuelve a encontrarse con Mr. Grimwig facilita su reconocimiento y refuerza su caracterización. En este capítulo, además, Dickens realza el carácter displicente del personaje a través del uso de 
growl para introducir sus palabras, como se puede comprobar en el siguiente ejemplo:

(6) 'You old women never believe anything but quack-doctors, and lying story-books,' growled Mr. Grimwig. 'I knew it all along. Why didn't you take my advise in the beginning; you would if he hadn't had a fever, I suppose, eh? He was interesting, wasn't he? Interesting! Bah!' And Mr. Grimwig poked the fire with a flourish. (Dickens, 2008 [1837-1839]: 137)

Por sí solas, las palabras de Mr. Grimwig no dejan entrever ningún rasgo de su carácter que ayude a definirle. Gracias a la utilización de growl para introducirlas, sin embargo, podemos percibir la mediación de Dickens a la hora de proyectar su irascibilidad ${ }^{7}$. Ahí es donde reside el potencial estilístico de los verbos de habla (Caldas-Coulthard, 1988: 6) y, en este caso concreto, el valor caracterizador de growl.

Tras estas apariciones en los capítulos 14 y 17, Mr. Grimwig desaparece de la historia durante veinticuatro capítulos. Como se ha comentado más arriba, esta ausencia alcanzó los once meses en la publicación original, pues la reaparición del personaje en el capítulo 41 corresponde a la decimoctava entrega, publicada en noviembre de 1838. Teniendo en cuenta la mencionada predilección de Dickens por hacer de sus figuras criaturas fácilmente reconocibles para el lector, no resulta sorprendente que en la reaparición del personaje Dickens recurra no solo a la muletilla, sino también a growl para introducir sus palabras, como se puede observar en el siguiente ejemplo:

(7) 'A bad one! l'll eat my head if he is not a bad one,' growled Mr. Grimwig, speaking by some ventriloquial power, without moving a muscle of his face. (Dickens, 2008 [1837-1839]: 331)

Cabe destacar que se trata nuevamente de la primera intervención del personaje en el capítulo, lo que refuerza la idea de que tanto la muletilla como growl son empleados por Dickens con el fin de evocar la imagen de Mr. Grimwig tras casi un año fuera de la trama. Esta intencionalidad queda demostrada si tenemos en cuenta que Dickens recurre a growl para modelar dos de sus actos

\footnotetext{
${ }^{7}$ Esta es la definición de growl como verbo de habla según el $O E D$ : b. 2. B trans. To utter or express with a growl or in a growling manner: with simple n., quoted words, or clause as obj. Su definición como sustantivo es la siguiente: 1. a. An act of growling; a low angry guttural sound uttered by an animal. [...] 2. 2 An expression of anger or dissatisfaction uttered by human beings.
} 
de habla justo a continuación y que en uno de ellos, además, inserta de nuevo la muletilla:

(8) 'Yes, he does,' growled Mr. Grimwig. (Dickens, 2008 [1837-1839]: 331)

(9) 'He'll eat his head, if he doesn't,' growled Mr. Grimwig. (Dickens, 2008 [1837-1839]: 331)

No cabe duda de que tanto el triple uso de growl como la doble utilización de la muletilla para construir las primeras intervenciones de Mr. Grimwig tras once meses ausente contribuyen a evocar su imagen, al tiempo que coadyuvan a reforzar su carácter irascible y desabrido.

Lo mismo ocurre, finalmente, cuando vuelve a aparecer por última vez en el capítulo 51. Desde su intervención en el capítulo 41 hasta su reaparición transcurren otros cuatro meses, tiempo suficiente para que Dickens estime oportuno volver a incidir en las particularidades discursivas del personaje cuando vuelve a hacer acto de presencia. Así, a pesar de que se trata del momento de júbilo y celebración con el que se cierra la historia y en el que por fin vemos la bondad de Mr. Grimwig, Dickens no desaprovecha la oportunidad, antes siquiera de que el personaje intervenga, de recordarnos que este, debido a su estado de felicidad, ya no amenaza con comerse la cabeza. He aquí el momento en el que el narrador hace mención a la marca discursiva del personaje, que ayuda a que lo identifiquemos rápidamente:

(10) [...] and here was Mr. Grimwig all ready to receive them, kissing the young lady, and the old one too, when they got out of the coach, as if he were the grandfather of the whole party, all smiles and kindness, and not offering to eat his head-no, not once; not even when he contradicted a very old postboy about the nearest road to London, and maintained he knew it best, though he had only come that way once, and that time fast asleep. There was dinner prepared, and there were bedrooms ready, and everything was arranged as if by magic. (Dickens, 2008 [1837-1839]: 417)

Sin embargo, este es un hábito tan característico del personaje que Dickens acaba incluyéndolo en uno de sus actos de habla. Lo hace en su última intervención en la novela. Aunque sea en tono de broma, el empleo de la muletilla contribuye a reforzar su caracterización en este último capítulo. He aquí el ejemplo: 
(11) 'I had serious thoughts of eating my head to-night,' said Mr. Grimwig, 'for I began to think I should get nothing else. I'll take the liberty, if you'll allow me, of saluting the bride that is to be.' (Dickens, 2008 [1837-1839]: 426)

Este uso de la muletilla en su última aparición en la historia sirve para cerrar el círculo de su caracterización. Desde el principio y hasta el final de su presencia textual, el personaje se caracteriza, además de por hablar en un tono gruñón, por una marca que lo individualiza y que, en gran medida, le otorga presencia en la historia. De no ser por esta habla idiosincrásica lograda a través de sus constantes amenazas con comerse la cabeza, Mr. Grimwig sería un personaje intranscendente en la novela. Gracias a esta particularidad, sin embargo, Dickens lo convierte no solo en un personaje fácilmente reconocible, sino en una figura con un alto grado de comicidad.

En suma, tanto el uso repetido de grow/ para introducir sus palabras como la propia muletilla atesoran un valor estilístico destacado en el marco de la caracterización de Mr. Grimwig. Además de definir al personaje y construirlo en el plano textual, Dickens emplea estos elementos para que el lector no tenga dificultades en reconocerlo tras ausencias que, como se ha podido comprobar, llegaron a alcanzar los once meses en la publicación original de la historia. Así pues, aunque las novelas de Dickens no hayan sido leídas habitualmente como trabajos publicados de manera fasciculada, este formato debe ser tenido en cuenta a la hora de analizarlas, pues se trata del soporte en el que desarrolló su producción y, sobre todo, uno de los aspectos que más influyó en su estilo (cf. Coolidge, 1967; Patten, 2000). Por ello, tanto el uso de la muletilla como la forma tan concreta de proyectar el discurso de Mr. Grimwig deberán pasar indemnes por el tamiz de la traducción, pues se corre el riesgo de anular una de las idiosincrasias estilísticas por antonomasia del autor.

\section{ANÁLISIS TRADUCTOLÓGICO}

En las versiones españolas de Oliver Twist de Alfredo Yáñez, Vergara y José Méndez Herrera se observa un grado de acierto desigual en el traslado de las particularidades del habla de Mr. Grimwig que acaban de ser descritas. A continuación se analiza la traducción por parte de los tres traductores de los ejemplos desglosados en el apartado anterior. El análisis traductológico se hará siguiendo un orden lineal, de tal suerte que pueda medirse el grado de acierto de las traducciones en el marco de la representatividad estilística de cada ejemplo en función del uso que Dickens hace de él en la novela original. 
En primer lugar, en cuanto a los dos usos de la muletilla empleados por Mr. Grimwig en sus dos primeras intervenciones en la historia y la explicación del narrador en la que nos informa de que se trata del hábito lingüístico más representativo del personaje (ejemplo (2) en el apartado 2.1.), el nivel de fidelidad por parte de los narradores es bastante dispar. He aquí, por un lado, la traducción de Yáñez:

(2a) - ¡Mire!, ¿no es cosa realmente prodigiosa que no pueda entrar yo aquí sin encontrar en la escalera una de estas cortezas de naranja que hacen la fortuna de los cirujanos? Una corteza de naranja me dejó cojo y otra me causará la muerte. ¡Sí, señor, una corteza de naranja causará mi muerte: apostaría la cabeza!

Ésta era la frase favorita de Mr. Grimwig para dar más expresión a lo que decía.

- Sí, señor, apostaría la cabeza —repitió Mr. Grimwig golpeando el suelo con su bastón-. ¡Hola!, ¿quién es éste? —añadió observando a Oliver y retrocediendo dos pasos. (Yáñez, 1996 [1945]: 106)

Como se puede advertir, Yáñez altera la muletilla de Mr. Grimwig, que es traducida como "apostarse la cabeza". Esta traducción, en principio, no parece demasiado acertada si la función de la marca discursiva del personaje es la de llamar la atención del lector y crear un elemento distintivo que sirva para reconocerle, pues "apostarse la cabeza" es una locución que existe en español ${ }^{8}$ y que, por tanto, no resulta sugerente como parte del idiolecto del personaje. De hecho, y quizá producto de la naturalidad de esta expresión en español, Yáñez no se detiene en la explicación del narrador salvo para informarnos de que se trata de su expresión preferida. "Apostarse la cabeza", por tanto, no parece una elección que vaya a quedar grabada en la retina del lector en este primer encuentro con el personaje.

Vergara, por su parte, traduce los dos primeros ejemplos de la muletilla de Mr. Grimwig en la novela y la explicación por parte del narrador del siguiente modo:

(2b) — ¡Qué bonito! ¿No es fatalidad y sino desgraciado que yo no pueda entrar en casa de un amigo sin tropezar en la escalera con una corteza de naranja, fortuna de los cirujanos? Otra pielecita de naranja me dejó ya cojo

${ }^{8}$ Esta es la definición de "apostarse la cabeza" según el Diccionario de la lengua española (Real Academia Española, 2014) (en adelante, $D R A E$ ): "apostarse la cabeza: 1. locs. verbs. U. para asegurar rotundamente lo que se dice". 
para toda la vida y estoy segurísimo de que otra pielecita será la causa de muerte. Sí, señor: a mí me matará una cáscara de naranja ¡Me comería la cabeza, señor!

Esta era la expresión favorita con que el señor Grimwig apoyaba sus asertos. Y era tanto más chusca esta frase en sus labios, cuanto que, aun admitiendo la posibilidad de tal disparate, la cabeza del señor Grimwig tenía mucho que comer por su excesivo tamaño, sin contar con que estaba excesivamente empolvada.

- Sí, señor, me comería la cabeza — repetía el rabioso viejo, golpeando el suelo con el bastón-. ¡Toma! ¿Qué significa esto? —añadió fijándose en Oliver y retrocediendo un paso. (Vergara, 1970 [1965]: 109-110)

La traducción de "me comería la cabeza", desde luego, resulta mucho más fiel al original. De un lado, mantiene la esencia dickensiana de la muletilla, a saber, dotar al personaje de una particularidad discursiva que llame la atención del lector cuando lo escucha por primera vez. De otro lado, además, la explicación del narrador abunda en la extrañeza de este hábito lingüístico. Esta explicación, como en el caso del texto original, se centra en lo improbable de que esto sucediera, lo que abunda en la comicidad de esta idiosincrasia discursiva del personaje y refuerza su carácter peculiar.

Méndez Herrera, por último, traslada este fragmento de texto como se muestra a continuación:

(2c) — ¡Mirad! ¿Veis esto? ¿No es verdaderamente maravilloso y extraño que no pueda visitar a nadie sin que me encuentre en la escalera con un trozo de este mísero amigo del cirujano? Me quedé cojo con una cáscara de naranja, y sé que, al final, una cáscara de naranja me costará la vida. De seguro, caballero; una cáscara de naranja será mi muerte. ¡Que me corten la cabeza si no, que me la como!

Era éste un magnífico ofrecimiento con el que el señor Grimwig apoyaba y confirmaba todos sus asertos, y bien singular por cierto, pues, aun admitiendo en hipótesis la posibilidad de que los adelantos científicos pudieran llegar a facultar a un hombre a comerse su propia cabeza, caso de que a ello estuviese dispuesto, la del señor Grimwig era tan descomunal, que ni el hombre más optimista podría abrigar la esperanza de terminar con ella de una sentada, dejando totalmente a un lado la cuestión de aquella espesa capa de polvos que la cubría.

- QQue me la como, sí, señor! —repitió Grimwig, dando un golpe en el suelo con un bastón-. ¡Ah, caramba! ¿Quién es éste? —añadió, fijándose en Oliverio y retrocediendo unos pasos. (Méndez Herrera, 2003 [1948]: 344345) 
Como se puede advertir, el traductor también mantiene la particularidad lingüística de Mr. Grimwig, haciéndola patente a través de una traducción fiel tanto de la propia muletilla como de la explicación del narrador. Es cierto que introduce un nuevo matiz, pues el personaje no solo amenaza con comerse la cabeza, sino que además pide que se la corten. Esta modificación, en cualquier caso, no supone una mella en la función estilística del hábito de Mr. Grimwig, pues sigue comportando una singularidad discursiva muy llamativa que individualiza al personaje a través de su habla y ayudará a evocar su imagen tras sus largas ausencias en la trama argumental.

En los dos casos restantes de la muletilla en el capítulo 14 (ejemplos (3) y (4) en el apartado 2.1.) observamos una línea continuista con respecto a los ejemplos que acaban de analizarse. Yáñez, por un lado, los traduce del siguiente modo:

(3a) — ¿Éste no será el muchacho que ha tenido la fiebre? - dijo Mr. Grimwig apartándose más-. ¡Espere usted! -añadió bruscamente, olvidando, sin duda, con la alegría de un descubrimiento el temor de contagiarse -; apuesto a que este muchacho es que ha pelado una naranja y ha tirado la corteza a la escalera. Apostaría la cabeza, y hasta las orejas. (Yáñez, 1996 [1945]: 106)

(4a) - Sí señor, lo dudo - repitió, descargando otro puñetazo sobre la mesa-, el muchacho lleva un vestido nuevo, debajo del brezo algunos libros de valor y en el bolsillo un billete de cinco libras esterlinas. Sin duda irá a dar un alegrón a sus antiguos compañeros, y todos juntos se burlarán de usted. Consiento en que me corten la cabeza si vuelve a poner los pies aquí. (Yáñez, 1996 [1945]: 110)

En el primero de ellos mantiene la locución española "apostarse la cabeza", que anula el despunte de la muletilla que ayuda a que identifiquemos al personaje. En el segundo ejemplo, además, sustituye la frase hecha por "que me corten la cabeza", rompiendo así con la consistencia de los usos anteriores y anulando el carácter sistemático del hábito lingüístico del personaje. Estas dos traducciones, por tanto, tampoco resultan atinadas para trasladar al español la particularidad discursiva del viejo solterón en su primera intervención en la historia, pues no logran crear el habla idiosincrásica que contribuye a individualizarlo en la novela.

Vergara, por su parte, traduce estos dos ejemplos de la siguiente forma: 
(3b) -No será el muchacho que ha padecido las fiebres, supongo exclamó el señor Grimwig retrocediendo asustado-. ¡Ah! -De pronto olvidó el temor al contagio ante la satisfacción del descubrimiento- ¡Ah! Apuesto a que ha sido este chico quien ha mondado la naranja y ha tirado la corteza por la escalera. ¡Me comería la cabeza y la suya también! (Vergara, 1970 [1965]: 110)

(4b) -Pues claro que lo dudo — dijo con el consabido puñetazo en la mesa-. El chico lleva un traje y zapatos nuevos, carios libros de precio bajo el brazo y un billete de cinco libras esterlinas en el bolsillo. Con todo eso se irá en busca de sus compañeros los ladrones y se burlará de ti. Si este chico pone aquí los pies otra vez, me como la cabeza. (Vergara, 1970 [1965]: 114-115)

De nuevo, al igual que en su traducción de los ejemplos iniciales, el traductor se muestra fiel al original y crea, en esta primera aparición del personaje en el capítulo 14, un personaje caracterizado, por encima de todo, por su idiosincrasia discursiva de comerse la cabeza.

Lo mismo ocurre, finalmente, con Méndez Herrera, pues en ambos amenaza nuevamente con comerse la cabeza, como se puede observar a continuación:

(3c) —No queréis decir que es el muchacho que ha estado con fiebre, ¿eh? —replicó el señor Grimwig, alegjándose un poco más-. ¡Esperad un instante! ¡No me digáis nada! ¡Deteneos!... —-continuó el señor Grimwig, perdiendo el miedo a la fiebre ante el triunfo del descubrimiento-. Este es el muchacho que ha pelado la naranja! Si no es este el que peló la naranja y arrojó el trozo de cáscara en la escalera, soy capaz de comerme mi cabeza, y la suya también. (Méndez Herrera, 2003 [1948]: 345)

(4c) -No - respondió, dejando caer el puño sobre la mesa-. No lo creo. El muchacho lleva sobre sus espaldas un traje nuevo, un montón de libros de valor debajo del brazo y un billete de cinco libras en el bolsillo. Irá en busca de sus antiguos amigos, los rateros, y se reirá de vos. Si alguna vez vuelve a esta casa ese muchacho, que me corten la cabeza, que me la como. (Méndez Herrera, 2003 [1948]: 348)

Cabe destacar que en el segundo de ellos, además, Mr. Grimwig vuelve a hacer referencia al matiz de que le corten la cabeza, al igual que hace en sus dos primeras intervenciones. Este matiz refuerza el componente cómico de la muletilla, que por su extrañeza ayudará a que identifiquemos rápidamente al personaje cuando vuelva a utilizarla más adelante. 
En segundo lugar, en cuanto a los ejemplos correspondientes al capítulo 17 -en los que Dickens recurre no solo a otro uso de la muletilla por parte de Grimwig (ejemplo (5) del apartado 2.1.), sino que además emplea growl por primera vez para introducir sus palabras (ejemplo (6)) -, la manera en que los traductores se enfrentan a la traducción de ambos recursos es de nuevo dispar, lo que afecta decisivamente a la caracterización del personaje. A continuación se muestra, en primer lugar, la traducción de la muletilla por parte de los tres traductores:

(5a) — ¿Un bedel! —exclamó Grimwig al divisar a Bumble_-, es un bedel de la parroquia; apuesto la cabeza. (Yáñez, 1996 [1945]: 131)

(5b) — ¡Un bedel! —exclamó el último apenas vio entrar al forastero-. Un conserje de hospicio. ¡Me comería la cabeza! (Vergara, 1970 [1965]: 140)

(5c) - iUn celador! ¡Si no es un celador parroquial, que me corten la cabeza, que me la como! (Méndez Herrera, 2003 [1948]: 364)

Yáñez, por un lado, mantiene la locución "apostarse la cabeza" que emplea para traducir los primeros ejemplos de la muletilla en la historia. Debido al uso extendido de esta expresión en español mencionado anteriormente, recurrir a ella para traducir la particularidad discursiva de Mr. Grimwig no parece la opción más acertada, sobre todo teniendo en cuenta que la función de este ejemplo de la muletilla en el texto original es eminentemente identificativa. En este sentido, las traducciones de Vergara y Méndez Herrera resultan mucho más certeras, pues emplean, como en el texto inglés, una construcción de carácter peculiar que contribuye a individualizar el discurso del personaje. Al tratarse de la primera intervención de Mr. Grimwig en el capítulo, estas dos traducciones contribuyen a evocar su imagen cuando vuelve a aparecer en la historia. Méndez Herrera, además, se muestra consistente en que el personaje pida que le "corten la cabeza" (2003 [1948]: 364) para que pueda comérsela, lo que aumenta el grado de individualización del hábito lingüístico y hace que despunte aún más como recurso de caracterización.

La traducción growl, por su parte, también presenta diferencias en las versiones españolas, como se puede comprobar a continuación:

(6a) -Ustedes, las viejas, no creen más que en los charlatanes y los cuentos - murmuró Grimwig-, pero hace ya mucho tiempo que yo sospechaba la verdad. ¿Por qué no haberme consultado desde un principio? Tal vez lo habría usted hecho, a no ser por la fiebre que le 
aquejaba, pero esto le hacía tan interesante, ¿no es cierto? ¡Qué lástima! (Yáñez, 1996 [1945]: 133)

(6b) - Las viejas no dan crédito sino a los charlatanes, ni escuchan más que los cuentos que hacen dormir en pie -murmuró el señor Grimwig-. Desde el principio supe yo a qué atenerme. ¡Como ustedes, si no hubiera sido por la fiebre! El pobrecito estaba malo. ¡Oh, qué dolor! ¡Qué lástima! (Vergara, 1970 [1965]: 142)

(6c) - Las viejas como vos no creen más que a los charlatanes y las mentiras de las noveluchas - refunfuñó el señor Grimwig-. Ya lo sabía yo. ¿Por qué no me pedisteis consejo desde un principio? Lo hubierais hecho, a no ser porque había tenido fiebre, ¿no es ese? Resultaba interesante, ¿verdad? ¡Interesante! ¡Bah!- y el señor Grimwig comenzó a atizar el fuego con ardor. (Méndez Herrera, 2003 [1948]: 365)

Tanto "murmurar"9 como "refunfuñar"10 denotan descontento en las palabras que introducen, al igual que el empleo de growl en el texto original. Sin embargo, ninguna de estas dos opciones conserva el tinte animalesco que sí atesora la forma verbal inglesa y que abunda en el carácter vehemente y exacerbado del viejo solterón. Quizás un verbo como "gruñir"11 habría resultado más acertado, pues tiene un cariz más exagerado que habría contribuido a caricaturizarlo. En cualquier caso, "murmurar" y "refunfuñar" conservan parcialmente el valor del verbo original y proyectan el carácter desabrido de Mr. Grimwig.

En tercer lugar, en lo que a la traducción de los ejemplos de la muletilla y del verbo de habla empleados en el capítulo 41 se refiere, mantener los matices originales resulta, si cabe, más importante. Como se ha señalado en el apartado 2.1, la ausencia del personaje entre los capítulos 17 y 41 alcanzó los once meses en la publicación original. Tras este lapso, Dickens hace uso tanto de la coletilla como del verbo de forma repetida (ejemplos (7) a (9) en el apartado 2.1) para evocar la imagen de Mr. Grimwig en su reaparición. De ahí la importancia de traducir estos ejemplos adecuadamente. En el caso de la primera intervención del personaje en el capítulo tras su larga ausencia, Dickens emplea la muletilla y el verbo de habla (ejemplo (7) en el apartado 2.1), lo que ayuda a que

${ }^{9}$ Esta es la definición de "murmurar" según el DRAE: "2. intr. Hablar entre dientes, manifestando queja o disgusto por algo. U. t. c. tr.".

${ }^{10}$ Esta es la definición de "refunfuñar" según el DRAE: "1. intr. Emitir voces confusas o palabras mal articuladas o entre dientes, en señal de enojo o desagrado".

11 He aquí la definición de "gruñir" según el DRAE: "2. intr. Mostrar disgusto y repugnancia, murmurando entre dientes". 
rememoremos, tras una ausencia de veinticuatro capítulos, su imagen displicente nada más oírle. De nuevo, en las tres versiones españolas se advierte un grado diferente de acierto, como se puede comprobar a continuación:

(7a) -Es un solemne tunante, apostaría a que es un solemne tunantemurmuró entre dientes Grimwig, impasible como un ventrílocuo. (Yáñez, 1996 [1945]: 310)

(7b) -Es un granujilla, me comería la cabeza si no es un granujilla murmuró entre dientes el señor Grimwig, tan impasible como un ventrílocuo. (Vergara, 1970 [1965]: 338)

(7c) — ¡Mala persona! Me apuesto la cabeza a que es una mala persona rezongó el señor Grimwig, hablando merced a alguna habilidad de ventrílocuo, sin mover un músculo de su cara. (Méndez Herrera, 2003 [1948]: 495)

La traducción de Yáñez es, en primer lugar, la menos acertada de las tres. Por un lado, recurrir al verbo "murmurar" solo mantiene parcialmente el valor de growl, pues, como se ha apuntado más arriba, conserva el desagrado de Mr. Grimwig en las palabras que pronuncia pero no proyecta el tinte animalesco de este. Por otro lado, en la traducción del acto de habla no se advierte rastro alguno de la muletilla. El simple uso de "apostaría" para traducir "I'll eat my head" anula el valor evocador que el hábito lingüístico atesora en el texto original y que Dickens decide colocar nuevamente en boca del personaje para que el lector pueda identificarlo rápidamente en su reaparición en la historia. Esta elección, por tanto, resulta errónea.

Por su parte, Vergara y Méndez Herrera, aunque mejoran la versión de Yáñez, muestran un grado de acierto desigual. Así, mientras que Vergara conserva la muletilla original intacta, Méndez Herrera se decanta, al igual que en los ejemplos de Yáñez comentados más arriba, por la locución española "apostarse la cabeza". Esta opción neutraliza la idiosincrasia discursiva del personaje, lo que dificulta su evocación tras once meses ausente de la trama. En la traducción del verbo de habla, sin embargo, Méndez Herrera se muestra más fiel al texto original que Vergara, pues "rezongar"12 conserva de un modo más fiel el tono desabrido y la actitud displicente de las palabras del personaje que "murmurar", opción menos marcada estilísticamente, como se acaba de señalar.

\footnotetext{
${ }^{12}$ Esta es la definición de "rezongar", según el DRAE: "1. intr. Gruñir, refunfuñar a lo que se manda, ejecutándolo de mala gana".
} 
En cuanto al siguiente uso de growl (ejemplo (8) en el apartado 2.1), empleado para introducir las palabras de Mr. Grimwig nuevamente tras apenas un par de intervenciones en el capítulo, también se observan diferencias en las tres traducciones. He aquí los ejemplos:

(8a) —iDe veras! —murmuró Mr. Grimwig. (Yáñez, 1996 [1945]: 310)

(8b) —Sí lo creo — gruñó Mr. Grimwig. (Vergara, 1970 [1965]: 338)

(8c) —Sí lo sabe — gruñó el señor Grimwig. (Méndez Herrera, 2003 [1948]:

495)

Como se puede advertir, Yáñez vuelve a recurrir a "murmurar" para reproducir el discurso del personaje. Esta elección resulta menos adecuada que la opción de "gruñir" escogida tanto por Vergara como por Méndez Herrera, con una carga de matices más cercana al verbo growl. "Gruñir" es, de hecho, la equivalencia ofrecida por los principales diccionarios bilingües inglés-español, como el de Oxford (McDermott, Goldsmith y Pérez Alonso, 1996), el de Collins (Smith, 1993) o el de Larousse (García Pelayo, 1991). Esta elección por parte de Vergara y Méndez Herrera, sin duda, resulta más acertada, pues mantiene el componente exagerado del verbo original, que contribuye a caricaturizar a $\mathrm{Mr}$. Grimwig y facilita su identificación en la historia.

El tercer ejemplo del capítulo 41, por último, es igualmente representativo, pues contiene de nuevo tanto la coletilla como el verbo de habla (ejemplo (9) en el apartado 2.1.). En este caso, como se puede comprobar a continuación, ninguna de las tres versiones conserva ambos rasgos de forma fiel:

(9a) —Apostaría a que sí lo sabe — volvió a replicar Grimwig. (Yáñez, 1996 [1945]: 310)

(9b) - Me comería la cabeza si él no lo cree también. (Vergara, 1970 [1965]: 338)

(9c) -Que se coma su cabeza si no lo sabe- insistió el señor Grimwig. (Méndez Herrera, 2003 [1948]: 495)

En la traducción de Alfredo Yáñez, en primer lugar, no se advierte rastro alguno de la idiosincrasia discursiva de Mr. Grimwig, pues recurre únicamente, como en su traducción del ejemplo anterior de la muletilla, a "apostaría". El hecho de que Yáñez no traduzca ninguno de los dos ejemplos de la muletilla en el capítulo 41 (ejemplos 7a y 9a) no solo dificulta la identificación del personaje por parte del lector tras veinticuatro capítulos ausente, sino que anula uno de los marchamos estilísticos dickensianos por excelencia, a saber, la utilización de la 
marca que caracteriza a un personaje cuando este vuelve a aparecer tras varias entregas ausente. Además, tampoco utiliza un verbo de habla específico para introducir las palabras de Mr. Grimwig del que se colija la irascibilidad de este. El empleo de "replicar"13, aunque refleja cierta renuencia por parte del personaje, no mantiene el tono gruñón ni el componente animalesco de growl que ayuda a caracterizarlo tras once meses fuera de la trama argumental.

Vergara, por su parte, sí que traduce adecuadamente la muletilla, incidiendo en la particularidad discursiva del personaje y facilitando su identificación por parte del lector tras su ausencia. Sin embargo, omite la forma verbal que introduce el acto de habla, pues transforma el ejemplo de estilo directo en un ejemplo de estilo directo libre, en el que las palabras del personaje no se encuentran introducidas por el narrador de manera explícita. Esta decisión incide directamente en la percepción del personaje por parte del lector, pues el verbo en el texto dickensiano, más que una función lingüística, atesora un valor estilístico que refuerza la imagen desabrida de Mr. Grimwig. La omisión del verbo de habla, por tanto, anula un recurso de caracterización clave empleado por Dickens para proyectar la imagen del personaje.

Méndez Herrera, por último, también se muestra fiel al original en la traducción de la muletilla, que nos ayuda a identificar al personaje a través de esta particularidad tras su ausencia. Es cierto, eso sí, que al contrario que en el texto inglés, no amenaza con comerse su propia cabeza, sino que dice que debe ser Oliver quien lo haga. Sin embargo, el componente identificativo se mantiene intacto, pues conserva el contenido original de la muletilla. En el caso del verbo de habla, sin embargo, su elección tampoco resulta acertada. De hecho, en el verbo "insistir"14 no se advierte ninguno de los matices que se proyectan con el uso growl comentados anteriormente y que apuntalan la caracterización de Mr. Grimwig, por lo que su uso también acarrea una pérdida significativa desde un punto de vista estilístico.

Finalmente, en el caso de los dos ejemplos de la muletilla correspondientes al capítulo 51, cuando el personaje ha desparecido nuevamente de la trama durante otros cuatro meses y Dickens vuelve a evocar su imagen a través del uso de su hábito lingüístico, en las traducciones también se advierten diferencias dignas de reseña desde un punto de vista estilístico. En primer lugar, en el caso del recordatorio del narrador sobre que Mr. Grimwig ya

\footnotetext{
${ }^{13}$ Esta es la definición de "replicar", según el DRAE: "2. intr. Responder oponiéndose a lo que se dice o manda. U. t. c. tr.".

${ }^{14}$ He aquí la definición de "insistir", según el DRAE: "1. intr. Instar reiteradamente".
} 
no articula la muletilla (ejemplo (10) en el apartado 2.1), los tres traductores se enfrentan a la traducción de diferente forma. He aquí los ejemplos:

(10a) [...] a la puerta encontraron a Grimwig dispuesto a recibir a los viajeros. Abrazó a Rose y a Mrs. Maylie apenas bajaron del coche, como si fuera su abuelo, y amable y complaciente, condujo a todos a las habitaciones interiores, donde encontraron la mesa servida y todo preparado, como por encanto, para recibirlos. (Yáñez, 1996 [1945]: 386)

(10b) [...] El señor Grimwig esperaba allí a los viajeros y salió a recibirlos; abrazó a la señorita y a la señora al bajar del coche como si él fuera el abuelo universal. Amable y sonriente, ni una vez intentó "comerse la cabeza" ni siquiera discutiendo con el viajo postillón que él conocía el mejor camino más corto a Londres, trayecto que sólo hiciera una vez y durmiendo todo el tiempo. La comida estaba servida, las habitaciones dispuestas, todo estaba preparado como por arte de magia para recibirlos. (Vergara, 1970 [1965]: 426)

(10c) [...] y allí estaba el señor Grimwig preparado para recibirlos, que besó a la joven, y a la vieja también, cuando salieron del coche, cual si fuera el abuelo de todos, lleno de sonrisas y amabilidades y sin que volviese a amenazar con comerse la cabeza ni una sola vez, ni siquiera cuando se puso a discutir con un viejo postillón sobre cuál era el camino más corto para ir a Londres, sosteniendo que él lo sabía mejor que nadie, a pesar de que sólo había seguido ese camino una sola vez y profundamente dormido. La cena estaba preparada, los dormitorios dispuestos y todo listo como por arte de magia. (Méndez Herrera, 2003 [1948]: 552)

Yáñez, siguiendo con la tónica de los últimos ejemplos analizados de sus traducciones de la muletilla, no mantiene la particularidad del personaje. De hecho, y quizás por no haberla traducido anteriormente, en este caso ni siquiera hace mención a ella. Naturalmente, esta omisión afecta a la caracterización del personaje, pues dificulta su reconocimiento por parte del lector tras diez capítulos fuera de la historia. Vergara, por su parte, sí que se muestra fiel al original y traduce adecuadamente la mención a la muletilla. Además, al ser el narrador quien nos informa en esta ocasión de la particularidad discursiva del personaje, decide entrecomillarla. De esta manera, incide en la idiosincrasia de Mr. Grimwig, lo que ayuda a reforzar el componente identificativo de la muletilla y contribuye a que el lector evoque su imagen tras su ausencia. Méndez Herrera, por último, también mantiene la mención a la particularidad de Mr. Grimwig por parte del narrador y traduce fielmente el texto original haciendo mención a la costumbre de 
amenazar con comerse la cabeza del personaje, lo que ayuda al lector a evocar la imagen del personaje en su reaparición.

Por otro lado, en la última ocasión en la que Grimwig pronuncia la muletilla (ejemplo (11) en el apartado 2.1.), que coincide con su última aparición en la historia, también observamos un grado de acierto desigual por parte de los traductores. He aquí las tres traducciones de la última intervención de Mr. Grimwig en la novela:

(11a) - Ya pensaba en comerme los codos a falta de otra cosa —exclamó Grimwig-. Me tomaré la libertad de ofrecer mis cumplidos a la futura de Harry. (Yáñez, 1996 [1945]: 396)

(11b) - Hoy sí que, en serio, pensaba comerme la cabeza... porque perdía la esperanza de comer otra cosa. $Y$ ahora, con vuestro permiso voy a presentar mis cumplidos a la novia. (Vergara, 1970 [1965]: 437)

(11c) - Ya empezaba a pensar seriamente que esta noche tendría que comerme la cabeza - dijo el señor Grimwig-, pues no creía contar con otra cosa. Me permitiréis que me tome la libertad de besar a la futura novia. (Méndez Herrera, 2003 [1948]: 559)

Como se puede advertir, Yáñez vuelve a fallar en la traducción del hábito lingüístico de Mr. Grimwig, pues "comerme los codos" no tiene nada que ver con su idiosincrasia discursiva. Esta traducción, unida a la omisión anterior en la explicación del narrador, neutraliza cualquier rastro de la particularidad que lo caracteriza en el capítulo 51, lo que anula el componente identificativo que esta desempeña. En el caso de las traducciones de Vergara y Méndez Herrera, por el contrario, sí que se mantiene la muletilla del personaje. Ambos traductores trasladan la marca discursiva de forma similar y logran retratar al personaje en su última intervención en la novela del mismo modo en que se le presenta nada más introducirlo, tal y como ocurre en el texto original. Su traducción, por tanto, sí que resulta acertada.

En definitiva, como se ha podido comprobar en el cotejo precedente, existen diferencias significativas en el grado de acierto de las versiones de Yáñez, Vergara y Méndez Herrera. Las traducciones de Vergara y Méndez Herrera muestran un nivel de refinamiento notablemente más alto que la de Alfredo Yáñez, en la que se han detectado numerosos errores. En el caso de la traducción de "I'll eat my head", por ejemplo, el hecho de que Yáñez no traduzca algunos de los ejemplos, recurra sistemáticamente a la comentada "apostarse la cabeza" en otros e incluso emplee en una ocasión "comerme los codos", no solo neutraliza el componente idiosincrásico de la muletilla del personaje en el texto 
original, sino que además anula la función caracterizadora que esta desempeña en el marco de la publicación fasciculada original de la novela. En su versión, al contrario que en las de Méndez Herrera y Vergara, se elimina un aspecto fundamental del estilo de Dickens.

La traducción de growl en las tres versiones, por su parte, merece un trato separado. Efectivamente, en ninguna de las tres traducciones se mantiene el componente colocacional del verbo. Esto es, ninguno de los traductores recurre a un único verbo para traducir los cuatro ejemplos de growl con los que Dickens introduce las palabras del personaje a lo largo de la historia y así dar lugar a un patrón que refuerce su carácter irascible. En cuanto a las opciones escogidas para trasladar el verbo original al español, cabe destacar la disparidad no solo entre las elecciones llevadas a cabo por los tres traductores, sino en los distintos verbos escogidos por cada uno de ellos para traducir los cuatro ejemplos de la forma verbal inglesa. Tanto la falta de homogeneidad como las habituales pérdidas de matices que se han detectado - ya sea a través de traducciones como replicar (Yáñez) o insistir (Méndez Herrera) o por la propia omisión del verbo (Vergara) ${ }^{15}$ - demuestran que los traductores no reparan en el valor estilístico de este elemento, que, como se ha visto, atesora un valor caracterizador y evocador en su disposición en el texto que sobrepasa la función lingüística -es decir, la de introducir discurso-que posee por definición.

En suma, las diferentes traducciones tanto de la muletilla como del verbo de habla por parte de los tres traductores han servido para ilustrar las implicaciones estilísticas que acarrean las elecciones más o menos acertadas en el traslado al español de estos elementos. En este sentido, todos los ejemplos, especialmente los menos atinados, evidencian la importancia de reparar en el valor literario de estos recursos empleados por Dickens para construir a sus personajes, pues su función caracterizadora - tanto en el plano estrictamente textual como en el marco de la publicación fasciculada original de sus novelaslos convierte en un aspecto fundamental de su estilo. Su correcto traslado al español, por tanto, debe ser una cuestión de primer orden en el proceso de traducción, pues, como se ha podido comprobar en el análisis que aquí se ha llevado a cabo, se corre el riesgo de anular una de las marcas por antonomasia de su estilo exagerado y caricaturesco.

${ }^{15}$ Véase la traducción del ejemplo (9). 


\section{CONCLUSIÓN}

Los personajes de las novelas de Dickens son acreedores de unas hablas muy peculiares que contribuyen a delinearlos como criaturas fácilmente reconocibles en el universo ficticio del autor. Esas hablas no solo son producto de las propias palabras que los personajes articulan, sino de la proyección de estas por parte del narrador. El ejemplo de Mr. Grimwig que aquí se ha analizado es un caso paradigmático en este sentido. Tanto sus constantes amenazas de comerse la cabeza como la proyección de su discurso a través de growl le dotan de un habla exagerada y con un alto componente caricaturesco que contribuye a modelar su carácter irascible y desabrido. Esta función caracterizadora del habla comporta un aspecto fundamental del estilo de Dickens, claramente influido por el marco de la publicación fasciculada de sus novelas. Como se ha visto, las intervenciones de Mr. Grimwig son muy intermitentes, con ausencias que en la publicación original alcanzaron los once meses. La individualización de su discurso a través de la muletilla y del verbo growl constituye un recurso de caracterización muy efectivo, pues ayuda a evocar la imagen del personaje tras sus significativas desapariciones de la trama argumental.

Desde el punto de vista de la traducción, la conservación tanto de la función caracterizadora a nivel puramente textual como del valor que estos elementos adquieren en el marco de la novela por entregas decimonónica inglesa debe ser una cuestión de primer orden. Sin embargo, ninguna de las traducciones que aquí se han analizado mantiene íntegramente el valor literario de estos recursos de caracterización. Principalmente en la versión de Yáñez, pero también en las de Vergara y Méndez Herrera, se han detectado pérdidas que acarrean una mella estilística con respecto al texto original. Estas pérdidas son más o menos significativas según el traductor y sobre todo según el ejemplo que se analice. En cualquier caso, todas afectan a la conservación de uno de los marchamos del autor victoriano, haciendo patente la necesidad de abordar la traducción de estos recursos desde un prisma estilístico más que desde un prisma exclusivamente comunicativo, pues en su traslado a otra lengua se está transfiriendo un aspecto clave de las técnicas de caracterización del autor y esencia misma de su estilo.

Cabe destacar, a guisa de cierre, que este artículo no ha pretendido menoscabar en modo alguno la labor de Yáñez, Vergara y Méndez Herrera como traductores. El fin último del estudio, por el contrario, ha sido el de poner de manifiesto la importancia de conservar aspectos clave de la novela victoriana en general y del estilo de Dickens en particular, aunque sin perder de vista las consabidas dificultades que comporta el siempre complejo arte de traducir. 
REFERENCIAS BIBLIOGRÁFICAS

BROOK, George Leslie, The Language of Dickens. London: Andre Deutsch, 1970.

BUSSE, Beatrix, Speech, Writing and Thought Presentation in a Corpus of Nineteenth-Century English Narrative Fiction. Bern. University of Bern, 2010.

CALDAS-CoulthaRd, Carmen Rosa, "Reported Speech in Written Narrative Texts". En: Discussing Discourse, Birmingham: University of Birmingham, 1987, pp. 149-167.

CoolidGE, Archibald C., Dickens as a Serial Novelist. lowa: lowa State University Press, 1967.

DICKENS, Charles, Oliver Twist. Oxford: Oxford University Press, 2008 [18371839].

FLUDERNIK, Monica, An Introduction to Narratology. London: Routledge, 2009.

HAWES, Donald, Who is Who in Dickens. London: Routledge, 2002.

García Pelayo, Ramón, Larousse Gran Diccionario. Español-Inglés. EnglishSpanish. Barcelona: Larousse, 1991.

Golding, R., Idiolects in Dickens: The Major Techniques and Chronological Development. London: Macmillan, 1985.

INGHAM, Patricia, "Speech and Non-Communication in Dombey and Son". En: The Review of English Studies, 30, 118, 1979, pp. 144-153.

MAHLBERG, Michaela, Corpus Stylistics and Dickens's Fiction. London: Routledge, 2013.

McDermott, Anella; Goldsmith, Patrick; Pérez Alonso, María Ángeles (eds.), Diccionario Oxford avanzado para estudiantes de inglés. Español-Inglés. Inglés-Español. Oxford: Oxford University Press, 1996.

MÉndez HeRRERA, José (trad.), Oliver Twist. Madrid: Aguilar, 2003 [1948].

PAGE, Norman, "Convention and Consistency in Dickens' Cockney Dialect". En: English Studies, 51, 1970, pp. 339-344.

PATTEN, Robert L., "Dickens as a Serial Author: A Case of Multiple Identities". En: Nineteenth-Century Media and the Construction of Identities, Basingstoke: Palgrave Macmillan, 2000, pp. 137-153.

Real Academia Española, Diccionario de la lengua española, vigesimotercera edición. Madrid: Espasa Calpe, 2014.

Ruano San Segundo, Pablo, "A Corpus-Stylistic Approach to Dickens' Use of Speech Verbs: Beyond Mere Reporting". En: Language and Literature, 25 (2), 2016, pp. 113-129.

SEMINO, Elena; SHORT, Mick, Speech, Writing and Thought Presentation in a Corpus of English Writing. London: Routledge, 2004. 
SмIтH, Colin, Collins Dictionary. Español-Inglés. Inglés-Español, tercera edición. Barcelona: Grijalbo, 1993.

VANN, J. Donn, Victorian Novels in Serials. New York: Modern Language Association, 1985.

VergaRA, L. (trad.), Oliver Twist. Barcelona: Círculo de Lectores, 1970 [1965].

YÁÑEZ, Alfredo (trad.), Oliver Twist. Barcelona: Océano, 1996 [1945]. 